\title{
Identification of tumor suppressive role of microRNA-132 and its target gene in tumorigenesis of prostate cancer
}

\author{
SHUN-LAI LI ${ }^{1}$, YING SUI ${ }^{1}$, JIE SUN ${ }^{2}$, TING-QI JIANG ${ }^{1}$ and GANG DONG ${ }^{1}$ \\ ${ }^{1}$ Department of Urology, The Fifth People's Hospital of Jinan, Jinan, Shandong 250012; \\ ${ }^{2}$ Tianqiao Hospital in Jinan of Shandong, Jinan, Shandong 250022, P.R. China
}

Received January 23, 2016; Accepted January 15, 2018

DOI: 10.3892/ijmm.2018.3421

\begin{abstract}
Previous literature exists on the role of microRNA (miR)-132 in initiation and progression of various malignancies. In this study, we aimed at understanding the relationship of miR-132 of prostate tumorigenesis. We collected 32 prostate cancer tissues and adjacent non-cancerous controls, and detected the expression level of miR-132. Then the miRNA database was searched online and luciferase assay perform to understand the regulatory relationship between miR-132 and E2F5. Moreover, we also conducted real-time PCR and western blot analysis to study the mRNA and protein expression level of E2F5 among different groups (cancerous tissue, $n=32$; non-cancerous tissue, $n=32$ ) or cells treated with scramble control, miR-132 mimics, E2F5 siRNA and miR-132 inhibitors. miR-132 was upregulated in cancerous tissues of prostate cancer patients. E2F5 was the target of miR-132, and negative regulatory relationship between miR-132 and E2F5 was also confirmed by luciferase assay. The mRNA and protein expression level of E2F5 increased in cancerous tissue group. miR-132 decreased the expression of E2F5 in prostate cancer cells, and introduction of miR-132 reduced the viability and E2F5 and promoted the viability of prostate cancer cells. miR-132 inhibited apoptosis and E2F5 accelerated apoptosis. In conclusion, miR-132 was upregulated in cancerous tissue of prostate cancer. E2F5 was a direct target of miR-132, and downregulation of E2F5 caused by upregulation of miR-132 may contribute to the tumorigenesis of prostate cancer.
\end{abstract}

\section{Introduction}

In the western world, prostate cancer is one of the most common cancers following skin cancer, with estimated one in nine men to have prostate cancer when they are at the

Correspondence to: Dr Jie Sun, Tianqiao Hospital in Jinan of Shandong, 25 Tianqiao Street, Tianqiao, Jinan, Shandong 250022, P.R. China

E-mail: prostatecancer@163.com

Key words: tumor suppressor, microRNA-132, E2F5, prostate cancer, apoptosis, tumorigenesis age of 75, and there are 20,000 cases being newly diagnosed in Australia each year, as published by the Prostate Cancer Foundation of Australia (information available at http://www. prostate.org.au, 2010). The well-proven risk factors for prostate cancer include race, age, and family history of prostate cancer (1). Moreover, considerable data show that genetic basis is a risk factor for prostate cancer $(2,3)$.

Previously, a microarray-based, genome-wide, comparative genomic hybridization analysis was performed to investigate the profiles of chromosomal changes in primary HCC and repeated amplification of the E2F5 gene harbored by the 8q21.2 locus in HCC was discovered (4). Representing the E2F transcription factor family, E2F5 binds to the promoters of the target genes associated with cell cycle control and subsequently has regulatory role in the expression of the target genes (5). Present downstream of the cascades of the growth factor signaling, the E2F family serves a crucial role in cell proliferation and growth by regulation of the genes associated with cell cycle progression (6). Hence, the members of the E2F family are likely to be implicated in oncogenesis. There are two subclassess of the members of the E2F family, including repressor (E2F4-E2F8) and activator (E2F1-E2F3) (5). It has been reported that overexpressed E2F activators trigger uncontrolled proliferated cells in a variety of human cancers including gastrointestinal, lung, ovarian and breast cancers (7-10). A significant quantity of studies support the possibility that some E2F repressors may act as oncogenes in tumorigenesis though the $\mathrm{E} 2 \mathrm{~F}$ repressors are considered to serve as tumor inhibitors (5).

Our understanding of the correlation between gene messenger RNAs (mRNAs) and human disease has changed and the discovery of miRNAs at the turn of the 21st century marked that cell biology had made a step into the new era, and since then has extended to the sequences in the residual $90 \%$ of eukaryotic genomes that produce non-coding RNAs. The microRNAs (miRNAs) play as meta-controllers of gene expression and are pivotal for the cellular alterations required for development (11). miRNA is an interesting and potential target to improve specificity of diagnosis because the expression of miRNA reveals the origin of the tumor and it has been associated with initiation and progression of prostate cancer (12-15). Currently, although there is no differential miRNA signature that enables to distinct healthy from disease patients, surprising findings have been derived for PCa. In a 
study of Volinia et al, the expression profile of 228 miRNAs was analyzed in 7 normal tissues and 56 prostate tumor tissues (16). Ambs et al conducted a study in a cohort of 76 micro-dissected tissues consisted of 16 controls and 60 tumor specimens and confirmed the upregulation of miR-93, miR-196a, miR-25, miR-92, miR-32, miR-26a, miR-181a, and let-7i (17). There was significantly upregulated expression of miR-101, miR-195, and miR-30c in patients who had extra-prostatic extension of cancer cells, indicating a potential role in prediction the progression of prostate cancer (18).

The data of miRNA microarray assay showed that miR-132 is dysregulated in prostate cancer compared with the control (19), indicating a role of miR-132 in the tumorigenesis of prostate cancer. Prediction algorithm was used to predict the target of miR-132, and based on the physiopathological functions of the virtual target genes obtained, we identified E2F5 in the followup study focusing on the reported involvement of E2F5 in the pathogenesis of prostate cancer (20). In this study, we validated E2F5 as a target of miR-132 and verified the involvement of miR-132 and E2F5 in the development of prostate cancer.

\section{Materials and methods}

Patients samples. Thirty-two prostate cancer patients who underwent surgery at the Tianqiao Hospital in Jinan of Shandong (Jinan, China) were recruited for this study between December 2013 and September 2014. The tumor sections as well as adjacent non-cancerous tissue were dissected. After resection, the specimens were immediately frozen in liquid nitrogen and then stored at $-80^{\circ} \mathrm{C}$. Participants or their first-degree relatives signed the informed consents before start of the experiment after careful explaination of all the potential risk factors. The Ethics Committee of The Tianqiao Hospital in Jinan of Shandong (Jinan, China) approved this study.

RNA isolation and real-time PCR. For analysis of the expression of E2F5 mRNA and miR-132 from tissue samples and LNCaP cells. TRIzol reagent (Invitrogen Life Technologies, Carlsbad, CA, USA) was used to extract the total RNA from LNCaP cells and tissue samples. And then oligo(dT) primer and SuperScript III reverse transcriptase (Invitrogen Life Technologies) were used to synthesize the cDNA. Mx3000P qPCR (Stratagene, La Jolla, CA, USA) was used to perform the quantitative reverse transcriptase polymerase chain reaction (qRT-PCR) with a mixture including 1X SYBR-Green $\mathrm{Tbr}$ polymerase (Finnzymes, Espoo, Finland), primers, 0.5X ROX. The thermal cycling was carried out one cycle for $10 \mathrm{~min}$ at $95^{\circ} \mathrm{C}$, followed by 45 cycles of $10 \mathrm{sec}$ at $95^{\circ} \mathrm{C}, 55^{\circ} \mathrm{C}$ for $30 \mathrm{sec}$ and $72^{\circ} \mathrm{C}$ for $30 \mathrm{sec}$. The $2^{-\Delta \Delta \mathrm{C}}$ method was used to analyze the relative quantification. The internal controls, GAPDH and RUN44, were used to control the expression of targets.

Cell culture and transfection. The LNCaP cells were prepared in RMPI-1640 medium including 10\% fetal bovine serum (FBS) (both from Gibco, Carlsbad, CA, USA) at $37^{\circ} \mathrm{C}$ in a humidified atmosphere of $5 \% \mathrm{CO}_{2} / 95 \%$ air. On transfection day, cells were cultured to $80 \%$, Lipofectamine 2000 (Invitrogen Life Technologies) was used for luciferase assay, transfection assay, apoptosis assay and proliferation assay in accordance with the manufacturer's instructions.
Cell proliferation assay. The cell proliferation reagent WST-1 (Roche Diagnostics, Indianapolis, IN, USA) was used to determine the growth of LNCaP cells based on the manufacturer's instructions. The optical density of the LNCaP cells was determined according to the absorption at $450 \mathrm{~nm}$ at different time-points using a microplate spectrophotometer (Tecan Group, Ltd., Männedorf, Switzerland). All experiments were repeated three times.

Luciferase assay. We search a public database (TargetScan, www.targetscan.org) for the target gene of miR-132 and E2F5 was found to contain the putative miR-132 binding site. PsiCHECK-2 reporter vector (Promega, Madison, WI, USA) was used to generate psiCHECK-2-E2F5-3'UTR by amplifying and inserting E2F5 3'UTR into the SpeI and HindIII sites of the empty vector and mutagenesis was performed for the same site, and introduced to the control vector (both from Ambion, Cambridgeshire, UK) at the same time. Lipofectamine 2000 (Invitrogen Life Technologies) was used in all the transfection assays in accordance with the manufacturer's instructions. Each test was repeated at least three times. Renilla luciferase activity was used as the internal control. Dual-Luciferase Reporter assay system (Promega, Massachusetts, MA, USA) was used to measure the activities of firefly and Renilla luciferase $48 \mathrm{~h}$ posttransfection. Three independent experiments were performed.

Western blot analysis. In order to detect the expression of E2F5 mRNA and miR-132, we collected the transfected cells, lysis buffer (BioSharp, Hefei, China) with $0.1 \%$ Triton X-100, $10 \mathrm{mmol} / 1$ sodium pyrophosphate, $2 \mathrm{mmol} / 1 \mathrm{EDTA}$, $50 \mathrm{mmol} / 1 \mathrm{NaF}$ and $150 \mathrm{mmol} / \mathrm{l} \mathrm{NaCl}$, to lyse the LNCaP cells. The cellular lysates were centrifuged at $15,000 \mathrm{rpm}$ for $15 \mathrm{~min}$. Approximately $35 \mu \mathrm{g}$ of protein was loaded using boiling water for 5 min with loading buffer. SDS-polyacrylamide gel (SDS-PAGE) (10\%) was used to separate the target protein, and then the protein was transfer-blotted onto polyvinylidene fluoride (PVDF) membrane (Millipore, Bedford, MA, USA). In order to avoid unspecific binding, the PVDF membranes were treated in the dark with $5 \%$ non-fat dried milk in TBST (BioSharp) $(150 \mathrm{mmol} / 1 \mathrm{NaCl}, 0.1 \%$ Tween-20 and $20 \mathrm{mmol} / \mathrm{l}$ Tris-HCl, $\mathrm{pH}$ 7.5). Then washed the membranes twice using TBST (BioSharp), next, treated with anti- $\beta$-actin antibodies (1:1,000; Sigma-Aldrich, St. Louis, MO, USA) and anti-E2F5 (1:1,000; Abcam, Cambridge, UK) for $12 \mathrm{~h}$ at $4^{\circ} \mathrm{C}$ in accordance with the manufacturer's instructions. The membranes were washed twice again using TBST (BioSharp), diluted HRP-conjugated anti-rabbit $\operatorname{IgG}(1: 3,000$; Abcam) to incubate the PVDF membrane at room temperature for $1 \mathrm{~h}$. The enhanced chemiluminescence system (Amersham Pharmacia Biotech, Braunschweig, Germany) was applied to detect the blots. The internal control was the expression of $\beta$-actin. Three independent tests were performed.

Analysis of apoptosis. The Annexin V-fluorescein isothiocyanate (FITC) Apoptosis Detection kit (C1062; Beyotime Institute of Biotechnology, Beijing, China) with Annexin V-FITC was used to stain the LNCaP cells according to the manufacturer's instructions. The flow cytometry (Cell Lab Quanta SC; Beckman Coulter Inc., Miami, FL, USA) with $585 / 42 \mathrm{~nm}$ PI and 530/30 nm FITC emission filters was used to 
detect the apoptosis of LNCaP cells. All tests were performed in triplicate.

Statistical analysis. SPSS statistical software (SPSS, Inc., Chicago, IL, USA) was used for statistical analysis. The Mann-Whitney U test was used to determine the statistical significance for continuous variables and the $\chi^{2}$ test or Fisher's exact test for categorical variables. P-values $<0.05$ were considered statistically significant. The data are shown as the mean \pm standard deviation (SD).

\section{Results}

miR-132 is upregulated in cancerous tissue of prostate cancer patients. We collected tumor tissue and adjacent non-cancerous tissue of prostate cancer patients $(n=32)$. Using real-time PCR, we found that the expression level of miR-132 was higher in cancerous tissue compared with the control (Fig. 1). The results indicated that miR-132 is negatively related to the tumorigenesis of prostate cancer.

E2F5 was a direct target of $m i R-132$. We first searched candidate target genes of miR-132 using publicly available databases, and consequently identified E2F5 as the candidate target gene with the potential binding site in the 3'UTR (Fig. 2). Thus, E2F5 was selected for further study. We conducted luciferase activity reporter assay in prostate cancer cells, and found that the fluorescence from the cells co-transfected with miR-132 and wild-type E2F5 3'UTR decreased significantly (Fig. 2), while cells co-transfected with miR-132 and mutant E2F5 3'UTR were comparable compared with scramble control (Fig. 3). The results confirmed that E2F5 was an immediate target of miR-132 in the cells.

Interaction between miR-132 and E2F5. To further clarify the role of miR-132 and E2F5 in tumorigenesis of prostate cancer, we collected 32 pairs of tissue samples (cancerous tissue, $n=32$; non-cancerous tissue, $n=32$ ). We first analyzed the correlation between the expression level of miR-132 and E2F5 mRNA among the tissues $(n=32)$, which showed negative regulatory relationship (Fig. 4; r=-0.556, p<0.05). We then performed real-time PCR, and found the expression of E2F5 mRNA (Fig. 5A) was increased in cancerous tissue compared with the control; the expression of E2F5 protein (Fig. 5B) was determined by densitometry analysis and it increased in cancerous tissue compared with chemotherapy sensitivity. These results suggested the presence of a negative regulator relationship between miR-132 and E2F5. To further verify the negative regulatory relationship between miR-132 and E2F5, we transfected the cells with scramble control, miR-132 mimics, E2F5 siRNA and miR-132 inhibitors. As shown in Fig. 6, overexpression of miR-132 decreased the protein (upper panel) and mRNA (lower panel) expression level of E2F5 compared with the scramble control, while downregulation of miR-132 increased the protein (upper panel) and mRNA (lower panel) expression level of E2F5, verifying the negative regulatory relationship between miR-132 and E2F5.

miR-132 and E2F5 interfere with the viability of prostate cancer cells. We also investigated the relative viability of cells

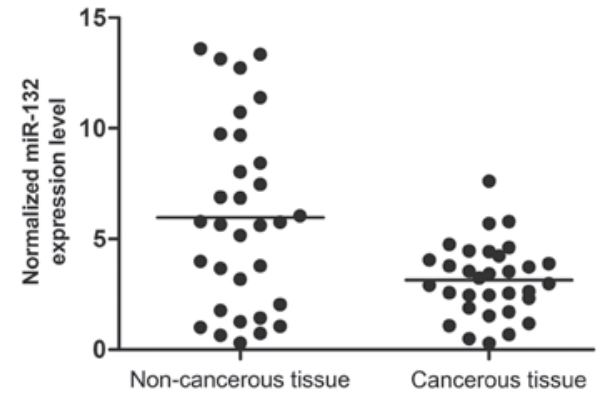

Figure 1. The expression level of microRNA (miR)-132 was higher in chemotherapy resistant group than chemotherapy sensitive group.

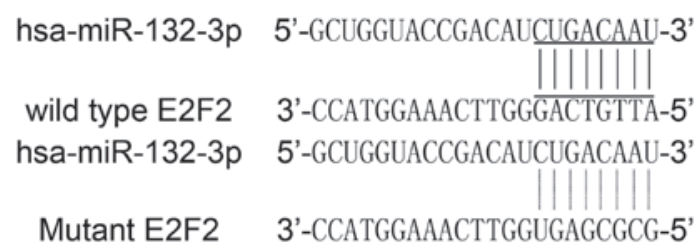

Figure 2. E2F2 as the candidate target gene of microRNA (miR)-132 in cells with the 'seed sequence' in the 3'UTR

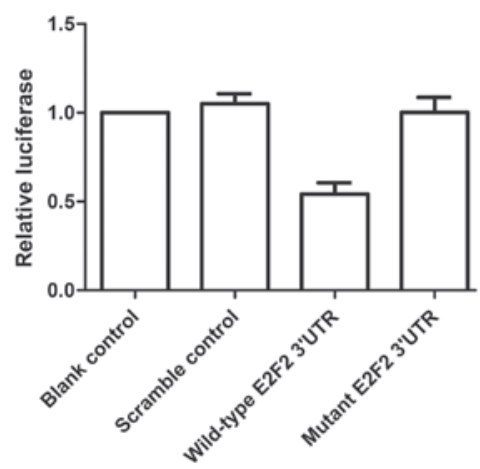

Figure 3. Luciferase activity reporter assay was conducted to verify E2F2 as the direct target gene of microRNA (miR)-132.

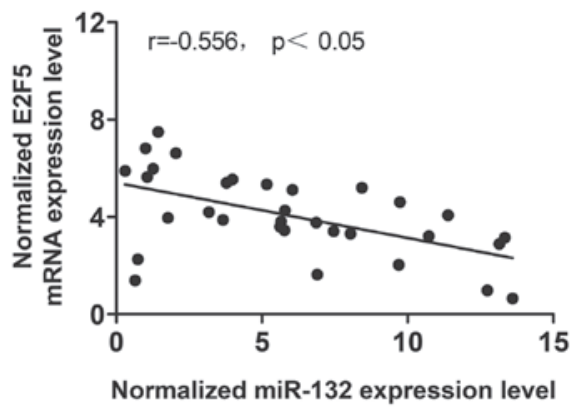

Figure 4. The correlation between the expression level of microRNA (miR)-132 and E2F2 messenger RNA (mRNA) between chemotherapy-sensitive and chemotherapy-resistant group (chemotherapy sensitive, $n=32$; chemotherapy resistant, $\mathrm{n}=32$ ).

when transfected with scramble control, miR-132 mimics, E2F5 siRNA and miR-132 inhibitors. As shown in Fig. 7A, downregulation of miR-132 attenuated viability of cells when compared with the scramble controls, while upregulation of miR-132 and downregulation of E2F5 enhanced viability, indi- 
A

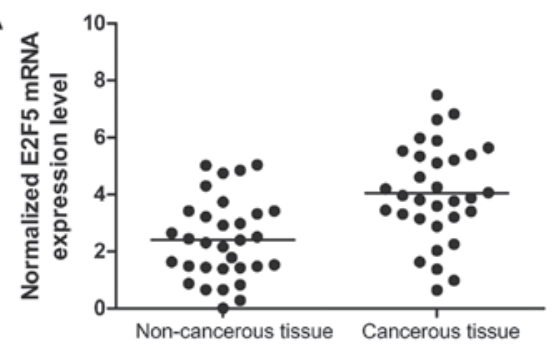

B

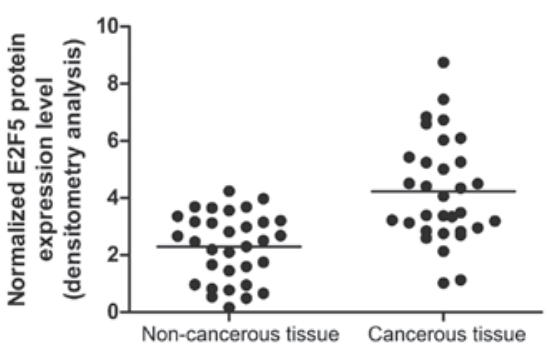

Figure 5. (A) The expression of E2F2 messenger RNA (mRNA) and (B) protein decreased in chemotherapy-resistant compared with chemotherapy-sensitive cells.

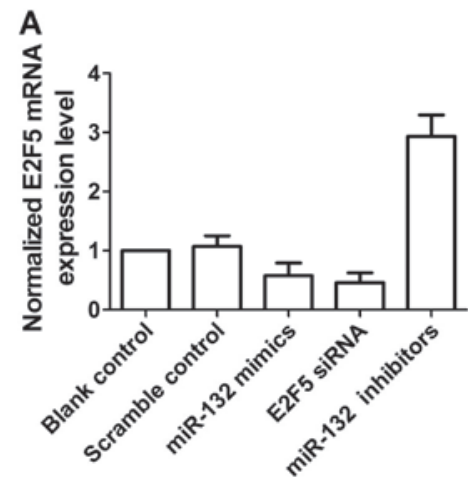

B

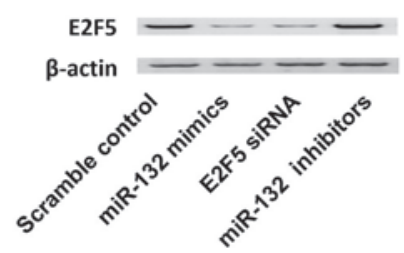

Figure 6. (A and B) When transfected with the cells with scramble control, microRNA (miR)-132 mimics, E2F2 siRNA and miR-132 inhibitors, the expression level of E2F2 protein (upper panel) and mRNA (lower panel) treated with miR-132 mimics and E2F2 siRNA decreased, while cells treated miR-132 inhibitors increased.

cating miR-132 positively interfered with the viability of cells, while E2F5 negatively interfered with the viability of cells.

miR-132 and E2F5 interfere with apoptosis in cells. We then investigated the relative apoptosis of cells when transfected with scramble control, miR-132 mimics, E2F5 siRNA and miR-132 inhibitors. When transfected with miR-132 mimics and E2F5 siRNA, the number of survival cells were more and the number of apoptotic cells were less than the scramble controls, while cells transfected with miR-132 inhibitors showed comparably less survival cells and more apoptotic cells. The results indicated miR-132 inhibited apoptosis and E2F5 accelerated apoptosis.
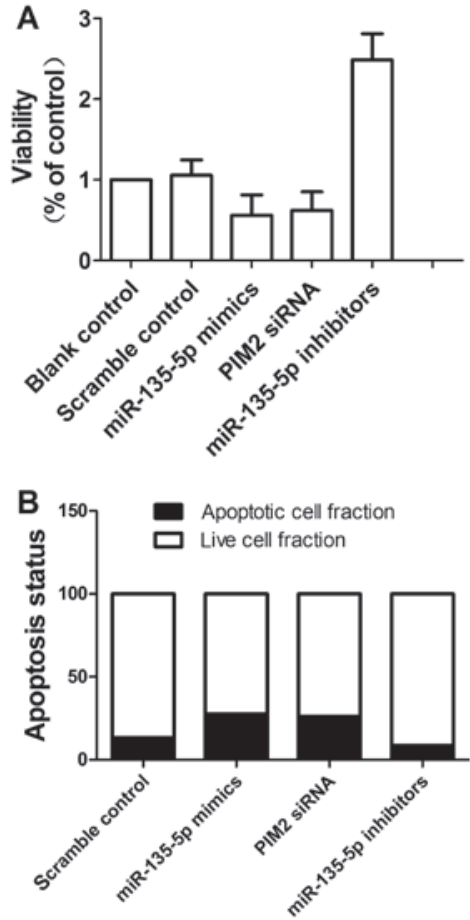

Figure 7. (A) Cells transfected with microRNA (miR)-132 inhibitors showed evidently downregulated viability, while cells transfected with miR-132 mimics and E2F2 siRNA showed comparably higher viability; (B) cells transfected with miR-132 mimics and MP2 siRNA, inhibited apoptosis while cells transfected with miR-132 inhibitors accelerated apoptosis.

\section{Discussion}

miR-132 is present in the intron of a non-coding gene on chromosome 17 in humans and originates from the miR-212/132 cluster (21). miR-132 is associated with angiotensin II (Ang II)-mediated dysfunction of the vascular smooth muscle, as demonstrated previously (22). In the tumorigenesis, the downregulated level of miR-132 prevents proliferation, migration, metastasis and invasion in breast cancer via affecting HN1 (23). Moreover, colorectal cancer metastasis and invasion are inhibited by miR-132 by affecting ZEB2 (24). Nevertheless, the mechanism by which miR-132 function as a regulator in tumor metastasis remains to be further investigated. In this study, we collected tumor tissue and adjacent non-cancerous tissue of prostate cancer patients $(n=32)$. Using real-time PCR, we found that the expression level of miR-132 was higher in cancerous tissue compared with the control (Fig. 1). In addition, we also performed real-time PCR, and found the expression of E2F5 mRNA (Fig. 5A) increased in cancerous tissue compared with the control; the expression of E2F5 protein (Fig. 5B) was determined by densitometry analysis and it increased in cancerous tissue compared with chemotherapy sensitive, indicating that the presence of a negative regulatory relationship between miR-132 and E2F5.

A conserved putative miR-132 target site in E2F5's 3'UTR was discovered by searching the online miRNA database. Subsequently, we conducted luciferase activity reporter assay in prostate cancer cells, and found that the fluorescence from the cells cotransfected with miR-132 and wild-type E2F5 3'UTR decreased significantly (Fig. 2), while cells cotransfected with miR-132 and mutant E2F5 3'UTR were comparable compared 
with scramble control (Fig. 3). We next analyzed the correlation between the expression level of miR-132 and E2F5 mRNA among the tissues $(n=32)$, which showed negative regulatory relationship (Fig. 4; r=-0.556, p<0.05).

Representing the E2F transcription factor family, E2F5 binds to the promoters of the target genes associated with cell cycle control and subsequently has regulatory role in the expression of the target genes (5). Present downstream of the cascades of the growth factor signaling, the E2F family serves a crucial role in cell proliferation and growth by regulation of the genes associated with cell cycle progression (6). Hence, the members of the E2F family are likely to be implicated in oncogenesis. There are two subclassess of the members of the E2F family, including repressor (E2F4-E2F8) and activator (E2F1-E2F3). It has been reported that overexpressed E2F activators trigger uncontrolled proliferation of cells in a variety of human cancers including gastrointestinal, lung, ovarian and breast cancers (7-10). A significant quantity of studies support the possibility that some E2F repressors may act as oncogenes in tumorigenesis though the E2F repressors are considered to serve as tumor inhibitors and although the E2F repressors are expected to behave as tumor suppressors (5). In this study, we investigated the relative viability of cells when transfected with scramble control, miR-132 mimics, E2F5 siRNA and miR-132 inhibitors. As shown in Fig. 7A, downregulation of miR-132 attenuated viability of cells when compared with the scramble controls, while upregulation of miR-132 and downregulation of E2F5 enhanced viability, indicating miR-132 positively interfered with the viability of cells, while E2F5 negatively interfered with the viability of cells.

We then investigated the relative apoptosis of cells when transfected with scramble control, miR-132 mimics, E2F5 siRNA and miR-132 inhibitors. When transfected with miR-132 mimics and E2F5 siRNA, the number of surviving cells are more and the number of apoptotic cells are less than the scramble controls, while cells transfected with miR-132 inhibitors showed comparably less surviving cells and more apoptotic cells. The results indicated miR-132 inhibited apoptosis and E2F5 accelerated apoptosis.

In conclusion, these findings indicate that E2F5 is a potential new target of miR-132 which is critical regulator in the development of prostate cancer, and reveals that E2F5 is a direct target gene of miR-132 although it is previously uncharacterized.

\section{Competing interests}

The authors declare there is no competing interest.

\section{References}

1. Crawford ED: Epidemiology of prostate cancer. Urology 62 (Suppl 1): 3-12, 2003.

2. Coughlin SS and Hall IJ: A review of genetic polymorphisms and prostate cancer risk. Ann Epidemiol 12: 182-196, 2002.

3. Witte JS: Prostate cancer genomics: towards a new understanding. Nat Rev Genet 10: 77-82, 2009.

4. Kim TM, Yim SH, Shin SH, Xu HD, Jung YC, Park CK, Choi JY, Park WS, Kwon MS, Fiegler H, et al: Clinical implication of recurrent copy number alterations in hepatocellular carcinoma and putative oncogenes in recurrent gains on 1q. Int J Cancer 123 2808-2815, 2008.

5. Chen HZ, Tsai SY and Leone G: Emerging roles of E2Fs in cancer: an exit from cell cycle control. Nat Rev Cancer 9: 785-797, 2009.
6. Ren B, Cam H, Takahashi Y, Volkert T, Terragni J, Young RA and Dynlacht BD: E2F integrates cell cycle progression with DNA repair, replication, and G(2)/M checkpoints. Genes Dev 16: 245-256, 2002

7. Han S, Park K, Bae BN, Kim KH, Kim HJ, Kim YD and Kim HY: E2F1 expression is related with the poor survival of lymph node-positive breast cancer patients treated with fluorouracil, doxorubicin and cyclophosphamide. Breast Cancer Res Treat 82: 11-16, 2003.

8. Reimer D, Sadr S, Wiedemair A, Stadlmann S, Concin N, Hofstetter G, Müller-Holzner E, Marth C and Zeimet AG: Clinical relevance of $\mathrm{E} 2 \mathrm{~F}$ family members in ovarian cancer - an evaluation in a training set of 77 patients. Clin Cancer Res 13: 144-151, 2007.

9. Eymin B, Gazzeri S, Brambilla C and Brambilla E: Distinct pattern of E2F1 expression in human lung tumours: E2F1 is upregulated in small cell lung carcinoma. Oncogene 20: 1678-1687, 2001

10. Lee J, Park CK, Park JO, Lim T, Park YS, Lim HY, Lee I, Sohn TS, Noh JH, Heo JS, et al: Impact of E2F-1 expression on clinical outcome of gastric adenocarcinoma patients with adjuvant chemoradiation therapy. Clin Cancer Res 14: 82-88, 2008.

11. Saugstad JA: MicroRNAs as effectors of brain function with roles in ischemia and injury, neuroprotection, and neurodegeneration. J Cereb Blood Flow Metab 30: 1564-1576, 2010.

12. Lu J, Getz G, Miska EA, Alvarez-Saavedra E, Lamb J, Peck D, Sweet-Cordero A, Ebert BL, Mak RH, Ferrando AA, et al: MicroRNA expression profiles classify human cancers. Nature 435: 834-838, 2005.

13. Coppola V, De Maria R and Bonci D: MicroRNAs and prostate cancer. Endocr Relat Cancer 17: F1-F17, 2010.

14. Fang YX and Gao WQ: Roles of microRNAs during prostatic tumorigenesis and tumor progression. Oncogene 33: 135-147, 2014.

15. Casanova-Salas I, Rubio-Briones J, Fernández-Serra A and López-Guerrero JA: miRNAs as biomarkers in prostate cancer. Clin Transl Oncol 14: 803-811, 2012.

16. Volinia S, Calin GA, Liu CG, Ambs S, Cimmino A, Petrocca F, Visone R, Iorio M, Roldo C, Ferracin M, et al: A microRNA expression signature of human solid tumors defines cancer gene targets. Proc Natl Acad Sci USA 103: 2257-2261, 2006.

17. Ambs S, Prueitt RL, Yi M, Hudson RS, Howe TM, Petrocca F, Wallace TA, Liu CG, Volinia S, Calin GA, et al: Genomic profiling of microRNA and messenger RNA reveals deregulated microRNA expression in prostate cancer. Cancer Res 68: 6162-6170, 2008.

18. Theodore SC, Davis M, Zhao F, Wang H, Chen D, Rhim J, Dean-Colomb W, Turner T, Ji W, Zeng G, et al: MicroRNA profiling of novel African American and Caucasian prostate cancer cell lines reveals a reciprocal regulatory relationship of miR-152 and DNA methyltranferase 1. Oncotarget 5: 3512-3525, 2014.

19. Zhao J, Wu XY, Ling XH, Lin ZY, Fu X, Deng YH, He HC and Zhong W: Analysis of genetic aberrations on chromosomal region 8q21-24 identifies E2F5 as an oncogene with copy number gain in prostate cancer. Med Oncol 30: 465, 2013.

20. Agarwal C, Dhanalakshmi S, Singh RP and Agarwal R: Inositol hexaphosphate inhibits growth and induces G1 arrest and apoptotic death of androgen-dependent human prostate carcinoma LNCaP cells. Neoplasia 6: 646-659, 2004.

21. Lau P, Bossers K, Janky R, Salta E, Frigerio CS, Barbash S, Rothman R, Sierksma AS, Thathiah A, Greenberg D, et al: Alteration of the microRNA network during the progression of Alzheimer's disease. EMBO Mol Med 5: 1613-1634, 2013.

22. Jin W, Reddy MA, Chen Z, Putta S, Lanting L, Kato M, Park JT, Chandra M, Wang C, Tangirala RK, et al: Small RNA sequencing reveals microRNAs that modulate angiotensin II effects in vascular smooth muscle cells. J Biol Chem 287: 15672-15683, 2012.

23. Zhang ZG, Chen WX, Wu YH, Liang HF and Zhang BX: miR-132 prohibits proliferation, invasion, migration, and metastasis in breast cancer by targeting HN1. Biochem Biophys Res Commun 454: 109-114, 2014.

24. Zheng YB, Luo HP, Shi Q, Hao ZN, Ding Y, Wang QS, Li SB, Xiao GC and Tong SL: miR-132 inhibits colorectal cancer invasion and metastasis via directly targeting ZEB2. World J Gastroenterol 20: 6515-6522, 2014. 